\title{
ON FINITE NUMBER OF CONJUGACY CLASSES IN GROUPS
}

\author{
JAN KREMPA, OLGA MACEDOŃSKA AND WITOLD TOMASZEWSKI
}

\begin{abstract}
The work is inspired by an article of M. Herzog, P. Longobardi, and M. Maj, who considered groups with a finite number of infinite conjugacy classes. Their main results were obtained under assumption that the $F C$-center is of finite index in the group. We consider here infinite groups with a finite number of conjugacy classes of any size ( $F N C C$-groups). Hence the $F C$-center in our case will be finite, but of infinite index in the group. Among results on these groups we give a criterion for a wreath product of $F N C C$-groups to be an $F N C C$-group.
\end{abstract}

\section{INTRODUCTION}

Many authors considered groups with some restrictions on conjugacy classes. Groups with conjugacy classes only of finite size, known as $F C$-groups, are well described e.g. in [3, 15, 17]. The generalization suggested in [8] releases definition of $F C$-groups by permitting a finite number of the infinite size conjugacy classes. In this paper we consider groups with a finite number of conjugacy classes of any size. They were called $C F$-groups in [11]. However, since ' $C F$ ' has many other meanings, we shall call these groups $F N C C$-groups.

Definition 1.1. A group is called FNCC-group if it has only Finite Number of Conjugacy Classes.

Clearly every finite group is an FNCC-group, while the infinite cyclic group is not an $F N C C$-group. The groups considered in [8], apart from Theorem 1.1(b), are $F N C C$-groups only in the case when they are finite. However, we are interested in infinite $F N C C$-groups.

The $F N C C$-groups, without special name, appear for example in [15, p. 129], [4, 5, 9], in [10, Problem 9.10] and in [14].

In 1949 the first example of an infinite $F N C C$-group was given in [9] by G. Higman, B. H. Neumann and H. Neumann. By means of the famous $H N N$-extension they proved that every torsion-free group can be embedded into a group with only two conjugacy classes. In 1952 this result was generalized by Yu. N. Gorchinskii, who gave a construction of groups with exactly $n$ conjugacy classes for every $n \geq 2[4$, Corollary

2010 Mathematics Subject Classification. Primary: 20E45, Secondary: 20E06, 20E22, 20F50.

Key words and phrases. conjugacy class, group extension, finiteness conditions. 
2]. However all these groups are infinitely generated, obtained as the unions of infinite sequences of $H N N$-extensions (or similar).

S. V. Ivanov proved (see [13] Thm. 41.2) that for any large enough prime $p$ there exists a 2-generator infinite group of exponent $p$ with exactly $p$ conjugacy classes, hence an $F N C C$-group.

The results of D. V. Osin from [14] imply that any countable group with only finitely many orders of elements can be embedded into a 2generator $F N C C$-group where any two elements of the same order are conjugate. This proves existence of finitely generated infinite $F N C C$ groups with $n(n \geq 2)$ conjugacy classes.

The importance of finitely generated $F N C C$-groups is shown in [11, Theorem 5.2] in connection with a criterion for a group of units in a group ring to be finitely generated. In the same paper it is shown, that every $F N C C$-subgroup of units in any associative ring with polynomial identity must be finite.

We describe here some properties of $F N C C$-groups concerning their subgroups, extensions and wreath products. Some results forcing finiteness of $F N C C$-groups will also be given. In this way we give a partial answer to Question 12 posed in [11].

Our notation will be rather standard, as for example in [16]. If $G$ is a group then for elements $a, b \in G$ we denote $[a, b]=a^{-1} b^{-1} a b=a^{-1} a^{b}$.

- $a^{G}$ denotes the conjugacy class of the element $a$ in the group $G$.

- $F C(G)$ - the $F C$-center of a group $G$, which consists of all elements $a \in G$ with $\left|a^{G}\right|<\infty$.

- $R(G)$ - the finite residual, which is the intersection of all subgroups of finite index in the group $G$.

- $C_{G}(R)$ - the centralizer of $R$ in the group $G$.

- A group is called anti-finite if it has no proper subgroups of finite index.

- A group is called locally graded if it has no finitely generated anti-finite subgroups.

\section{BASIC PROPERTIES OF FNCC-GROUPS}

Certainly the class of all FNCC-groups is closed under homomorphisms and finite direct products.

Lemma 2.1. Let $G$ be an FNCC-group with $k$ conjugacy classes and let $R \subseteq G$ be a subgroup of index $m<\infty$. Then

(i) $G$ is a union of $n$ conjugacy classes with respect to $R$, where $n \leq k m$

(ii) $R$ is an FNCC-group. 
Proof. (1) By assumptions we have $G=\bigcup_{i=1}^{k} g_{i}^{G}=\bigcup_{j=1}^{m} b_{j} R, g_{i}, b_{j} \in$ $G$,

Then $\quad G=\bigcup_{i=1}^{k} \bigcup_{j=1}^{m} g_{i}^{\left(b_{j} R\right)}=\bigcup_{i=1}^{k} \bigcup_{j=1}^{m}\left(g_{i}^{b_{j}}\right)^{R}=: \bigcup_{i=1}^{n} a_{i}^{R}$, where $a_{i} \in G$.

(2) If $a_{i}^{R} \cap R \neq \emptyset$, then $a_{i}^{R} \subseteq R$. This, together with (i), implies that $R$ is an $F N C C$-group.

The properties considered in the following Lemma are addressed below only by their numbers.

Lemma 2.2. Let $G$ be an FNCC-group with the finite residual $R:=$ $R(G)$ and with the $F C$-center $F:=F(G)$. Then:

(i) $R$ is of finite index in $G$;

(ii) $F$ is finite;

(iii) $R$ is an anti-finite FNCC-group;

(iv) The centralizer of $R$ in $G$ is equal to $F$;

(v) If $G$ is infinite, then $R$ has a simple infinite homomorphic image;

(vi) If $G$ is torsion, then it is a group of finite exponent.

Proof. (i) Each $F N C C$-group has only finite number of normal subgroups, hence $R$ is of finite index in $G$.

(ii) The property follows immediately from the definition of $F N C C$ group.

(iii) By $(i)$ and the definition of $R$, it has no proper subgroup of finite index, thus it is anti-finite. Lemma 2.1(ii), gives that $R$ is an $F N C C$-group.

(iv) Note first that the centralizer $C_{G}(F)=\cap_{f \in F} C_{G}(f)$ is, by $(i i)$, a subgroup of finite index in $G$. Then $C_{G}(F)$ contains $R$ and hence $C_{G}(R) \supseteq F$. For the converse inclusion we take $a \in C_{G}(R)$, then $C_{G}(a) \supseteq R$ and $\left|a^{G}\right|=\left|G: C_{G}(a)\right| \leq|G: R|$. Since by $(i)|G: R|<\infty$ we get $a \in F$, that is $C_{G}(R) \subseteq F$. The equality follows.

(v) If $G$ is infinite, then by $(i), R$ is infinite and by $(i i i), R$ is an antifinite $F N C C$-group. Hence $R$ contains a maximal normal subgroup $N$, and $R / N$ is infinite simple.

(vi) An $F N C C$-group has only finite number of orders of elements and being torsion, it has a finite exponent.

By property $(v i)$ of the above Lemma we have that every torsion $F N C C$-group satisfies a law of the form $x^{n} \equiv 1$ for some $n \geq 1$. This is an example of so called $\mathfrak{R}$-law. Recall that a law $w \equiv 1$ is an $\mathfrak{R}$-law if every finitely generated group $G$ satisfying this law has $G^{\prime}$ finitely generated, (see [12, Definition 6.2]). Positive laws and Engel laws are R-laws (see [12, Corollary 6.4]). 
The following Theorem generalizes Proposition 5.5 in [11] and contains Theorem 5.6 from that paper.

Theorem 2.3. If $G$ is an FNCC-group, then $G$ is finite in any of the following cases:

(a) $G$ is linear over a field;

(b) $G$ is locally or residually finite;

(c) $G$ is locally or residually soluble;

(d) $G$ is locally graded and either is finitely generated, or satisfies an $\mathfrak{R}$-law, or is torsion.

Proof. (a) This proof, based on classical arguments of Burnside, is more detailed than that in [11]. Let $G \subseteq G L(n, K)$ be an $F N C C$-group. The field $K$ can be assumed algebraically closed. We proceed by induction on $n$. If $n=1$ then $G$ is abelian, hence finite.

Let $n>1$. Assume first, that $G$ is irreducible as the group of linear transformations of $K^{n}$. Since $G$ is an $F N C C$-group, the set of traces of all elements of $G$ is finite, because conjugate matrices have the same traces. Hence by [7, Theorem 2.3.3], $G$ is finite.

Now let $G \subseteq G L(n, K)$ be reducible. After proper choice of a base in $K^{n}$ we can assume that there exists $m, 1<m<n$, such that every $g \in G$ is of the form $\left[\begin{array}{cc}\alpha(g) & x \\ 0 & \beta(g)\end{array}\right]$, where $\alpha(g) \in G L(m, K)$ and $\beta(g) \in G L(n-m, K)$. The functions $\alpha$ and $\beta$ are homomorphisms of groups. Thus $\alpha(G)$ and $\beta(G)$ are $F N C C$-groups, and hence finite, by the inductive assumption. Let $N$ be the intersection of kernels of $\alpha$ and $\beta$. Then $G / N$ is finite and $N$ is an $F N C C$-group, by Lemma 2.1(ii), as a subgroup of finite index in $G$. On the other hand, $N$ is soluble, and even nilpotent, as a subgroup of unitriangular matrices of the form $\left[\begin{array}{cc}1_{m} & x \\ 0 & 1_{n-m}\end{array}\right]$. Hence $N$ and so $G$ are finite, which proves (a).

In the text below, we shall assume the contrary, that $G$ is an infinite $F N C C$-group and show, that it leads to a contradiction.

(b) Let $G$ be locally finite. By $(v i) G$ is of finite exponent, because it is torsion. By $(v)$, we can assume that $G$ is simple. Hence, (compare [2, Lemma 4]) $G$ does not involve all finite groups, and then by [6, Theorem 2.6], it is linear. Now by $(a), G$ is finite. A contradiction.

Since $G$ is infinite, by $(i), R(G)$ is nontrivial. Hence $G$ cannot be residually finite.

(c) Let $G$ be locally soluble. By $(v)$, we can also assume that $G$ is an infinite simple group. However by ([16], 12.5.2) such a group is finite. A contradiction.

Let $G$ be residually soluble. Since $G$ has only finite number of normal subgroups, and each soluble quotient of $G$ is finite (which is proved just above), we conclude that $G$ is finite. A contradiction.

(d) Let $G$ be locally graded. If $G$ is finitely generated $F N C C$-group then its finite residual $R$ has finite index, so is finitely generated. Since 
$G$ is locally graded, $R$ has a subgroup of finite index, which is impossible, because by (iii) $R$ is anti-finite.

If $G$ satisfies an $\mathfrak{R}$-law then by [12, Corollary 6.8$]$ based on [1, Theorem B], $G$ is nilpotent-by-locally finite. Hence, being an FNCC-group, $G$ is by (b), nilpotent-by-finite. By Lemma 2.1(ii) and (c), the nilpotent normal subgroup of finite index in $G$ is finite. Hence $G$ is finite. A contradiction.

If $G$ is torsion then by $(v i)$ it has a finite exponent. Then it satisfies an $\mathfrak{R}$-law and, by the above case, is finite. A contradiction.

In connection with the above properties the following question arises.

Question 2.4. Does there exist an infinite and locally graded FNCCgroup?

In view of the above theorem it suffices to look for an infinitely generated locally graded $F N C C$-group without subgroups of finite index. Moreover, such a group, if exists, could not be periodic. In the previous section we indicated the existence of infinite simple $F N C C$-groups. Some of them are certainly not locally graded.

As a consequence of Lemma 2.2 we obtain

Theorem 2.5. An infinite FNCC-group $G$ has a normal series

$$
G \triangleright R \triangleright Z(R) \text {, where } R:=R(G) .
$$

For this series we have

- $G / R$ is finite,

- $R / Z(R)$ is anti-finite FNCC-group with trivial FC-center,

- $Z(R)=C(R) \cap R=F C(G) \cap R$ is finite abelian.

Proof. In view of Properties (i), (iii), G/R is finite, and $R / Z(R)$ is anti-finite $F N C C$-group. We show now that $Z(R)=F C(R)$.

Let $a \in F C(R)$, then $\left[R: C_{R}(a)\right]<\infty$. However, by (iii), $R$ is antifinite and it follows that $a \in Z(R)$. This means that $F C(R) \subseteq Z(R)$. The converse inclusion is clear, so $F C(R)=Z(R)$. Since $R$ is $F N C C$ group, $F C(R)=Z(R)$ is finite abelian. The quotient $R / Z(R)$ is an anti-finite $F N C C$-group, which implies (as above) that its center and $F C$-center coincide. Since by $(i v), F C(R)$ is finite, the $F C$-center of $R / Z(R)$ is trivial.

Question 2.6. Does there exist an anti-finite FNCC-group $G$ with $Z(G) \neq 1$ ?

\section{EXTENSIONS}

The question whether the class of $F N C C$-groups is closed for extensions is still open, however in some special cases we can give a positive answer. To give an answer for finite-by- $F N C C$-groups, we first prove an auxiliary result 
Lemma 3.1. Let $N$ be a finite normal subgroup in a group $G$. If $G / N$ is an $F N C C$-group, then the finite residual $R(G)$ is of finite index in $G$, and the $F C$-center $F C(G)$ is finite.

Proof. Let $G / N$ be an FNCC-group. Then, by property $(i), R(G / N)$ is of finite index in $G / N$. Let $R(G / N)=H / N$, where $N \subseteq H$. Then $|G: H|<\infty$, and hence $R \subseteq H$. On the other hand, if $X \triangleleft G$ and $|G: X|<\infty$, then also $|G: N X|<\infty$, and hence $H / N=$ $R(G / N) \subseteq N X / N$. In this way we obtain that $H \subseteq N X$. Hence, $|G / X|=|G / N X||N X / X| \leq|G / H| \cdot|N|$, which is a common bound for indices of subgroups $X$ of finite index in $G$. Now by Third Isomorphism Theorem $|G / R|<\infty$, so $R(G)$ is of finite index in $G$.

Since $G / N$ is $F N C C$-group, we have by $(i i)$, that $F C(G / N)$ is finite. The assumption $|N|<\infty$ implies $N \subseteq F C(G)$. Then $F C(G) / N=$ $F C(G / N)$ is finite and hence $F C(G)$ is finite.

Theorem 3.2. If $N$ is a finite normal subgroup in a group $G$ and $G / N$ is an FNCC-group, then $G$ is an FNCC-group.

Proof. Let $|N|=n$ and $G / N$ be an FNCC-group. By assumption $G / N$ is a sum of say $s$ conjugacy classes, then for some $a_{i} \in G$

$$
G=a_{1}^{G} N \cup a_{2}^{G} N \cup, \ldots \cup a_{s}^{G} N .
$$

By Lemma 3.1, $R(G)$ has a finite index $l$, say, in $G$, hence

$$
G=g_{1} R \cup g_{2} R \cup, \ldots g_{l} R, \quad g_{i} \in G .
$$

By Lemma 3.1, $F C(G)$ is finite. Similarly as in the proof of $(i v)$ we can get that $R(G)$ centralizes $F C(G)$ and hence $F C(G)$ centralizes $R(G)$. The assumption that $|N|<\infty$ implies $N \subseteq F C(G)$, thus we obtain that $N$ centralizes $R(G)$,

$$
N \subseteq C_{G}(R) .
$$

We have three sets of elements:

$$
\left\{a_{i}, i=1,2, \ldots s\right\}, \quad\left\{g_{j}, j=1,2, \ldots l\right\}, \quad N=\left\{x_{k}, k=1,2, \ldots, n,\right\},
$$

and show that $G$ is a sum of a finite number of conjugacy classes $\left(a_{i}^{g_{j}} x_{k}\right)^{G}$. It suffices to check that each element in $G$ is in such a class. Let $b \in G$, then there is $a_{i}, g \in G$ and $x_{k} \in N$ such that $b=a_{i}^{g} x_{k}$. Moreover, there is $g_{j} \in G$ such that $g \in g_{j} R$. Then since $N \subseteq C_{G}(R)$,

$$
b=a_{i}^{g} x_{k} \in a_{i}^{g_{j} R} x_{k} \subseteq\left(a_{i}^{g_{j}} x_{k}\right)^{R} \subseteq\left(a_{i}^{g_{j}} x_{k}\right)^{G} .
$$

Hence $G$ is an $F N C C$-group with no more then sln conjugacy classes.

Theorem 3.3. Let $R$ be a normal subgroup of finite index in a group $G$. If $R$ is an FNCC-group and every inner automorphism of $G$ restricted to $R$ is an inner automorphism of $R$, then $G$ is an FNCC-group. 
Proof. Let $\varphi: G \rightarrow A u t(R)$ be a homomorphism given by

$$
\varphi(g): r \rightarrow r^{g} \quad \text { for } \quad g \in G \text { and } r \in R .
$$

From the assumption on automorphisms we have that $\varphi(G)=\varphi(R)$ is an FNCC-group, as a homomorphic image of $R$. The kernel of $\varphi$ is equal to $C=C_{G}(R)$. The subgroup $C \cap R$ is finite, because $R$ is an $F N C C$-group. The group $C /(C \cap R) \simeq C R / R \subseteq G / R$ is finite, by assumption on $R$. Thus $C$ is finite. Now, $G / C \simeq \varphi(G)$ is an $F N C C$ group and hence, by Theorem 3.2, $G$ is an $F N C C$-group.

The following question is natural is this place

Question 3.4. Let $R$ be a normal subgroup of finite index in a group $G$. Assume that $R$ is an FNCC-group and every inner automorphism of $G$ restricted to $R$ preserves conjugacy classes in $R$. Is $G$ an FNCCgroup?

Now we show that to speak of a finite extension of an FNCC group, it suffices to consider only finite cyclic extensions.

Lemma 3.5. Let $G$ be a group and let $G=\bigcup_{j=1}^{m} G_{j}$, where $G_{j} \subseteq G$ for $j=1, \ldots, m$. If the subgroups $G_{j}$ are $F N C C$-groups then $G$ is an FNCC-group.

Proof. Let each $G_{j}$ be an $F N C C$-group. By assumption there are elements $a_{j 1}, a_{j 2}, \ldots, a_{j n_{j}} \in G_{j}$ such that

$$
G_{j}=\bigcup_{i=1}^{n_{j}}\left(a_{j i}\right)^{G_{j}}, \quad \text { which implies } G=\bigcup_{j=1}^{m} \bigcup_{i=1}^{n_{j}}\left(a_{j i}\right)^{G} \text {, }
$$

and hence $G$ is an $F N C C$-group.

Let $R \subseteq G$ be a normal subgroup of finite index. Then there are elements $a_{1}, \ldots, a_{n} \in G$ such that

$$
G=\bigcup_{i=1}^{n}\left\langle R, a_{i}\right\rangle=\bigcup_{i=1}^{n} G_{i}, \quad \text { where } G_{i}=\left\langle R, a_{i}\right\rangle .
$$

Now for $G_{i}$ we have a number $l_{i}$ and a normal series

$$
R=H_{i 0} \leq \ldots \leq H_{i l_{i}}=G_{i}
$$

such that all factor groups $H_{i j} / H_{i(j-1)}$ are cyclic of prime orders.

Under notation from (1) and (2) in Lemma 3.5 we have

Corollary 3.6. Let $R \subseteq G$ be a normal subgroup of finite index. If $R$ is an FNCC-group then $G$ is an FNCC-group if and only if groups $\left\langle R, a_{i}\right\rangle$ are FNCC-groups, or equivalently, groups $H_{i j}$ are FNCCgroups. 
Remark. If one is interested only in semidirect product of the type $G=R \rtimes F$, where $F$ is a finite group then, by (1), it suffices to consider only the case when $F$ is cyclic, hence it is a direct product of cyclic $p$-groups. Thus one can restrict to the case when $F$ is cyclic of prime power order, because for $F=F_{1} \times F_{2}$ we have in a natural way the following formula:

$$
R \rtimes\left(F_{1} \times F_{2}\right) \simeq\left(R \rtimes F_{1}\right) \rtimes F_{2} .
$$

Now we concentrate on a special type of semidirect products.

Lemma 3.7. Let $P$ be an FNCC-group and $R=\prod_{i}^{n}{ }^{\times} P_{i}$, - the direct product of $n$ copies of $P$. Let $\langle b\rangle_{n}$ be a cyclic group of order $n$, and $G=R \rtimes\langle b\rangle_{n}$ - the semidirect product, where $b$ acts on $R$ as a cyclic permutation of factors. Then the coset $b R$ is contained in a union of finite number of conjugacy classes in $G$.

Proof. The set of representatives of conjugacy classes in $P$ we denote by $\mathcal{P}:=\left\{p_{1}, p_{2}, \ldots, p_{m}\right\}$. Then the elements in $R$ are of the form

$$
\left(p_{j_{1}}^{a_{1}}, p_{j_{2}}^{a_{2}}, \ldots, p_{j_{n}}^{a_{n}}\right)=\left(p_{j_{1}}, p_{j_{2}}, \ldots, p_{j_{n}}\right)^{\left(a_{1}, a_{2}, \ldots, a_{n}\right)}, \quad p_{j} \in \mathcal{P}, \quad a_{j} \in R .
$$

Hence $R$ has $m^{n}$ conjugacy classes with the representatives

$$
\rho=\left(p_{j_{1}}, p_{j_{2}}, \ldots, p_{j_{n}}\right), \quad p_{j} \in \mathcal{P} .
$$

To prove the Lemma we show that each element $b r \in b R$, is in some of $m^{n}$ conjugacy classes of the form $\left(b \rho_{z}\right)^{G}$. It suffices to find for each $r \in R$ such $x \in R$ and $\rho_{z}$ of the form (5), that the following equality holds $b r=\left(b \rho_{z}\right)^{x}$. In view of the identity

$$
(b \rho)^{x}=x^{-1}(b \rho) x=b b^{-1} x^{-1}(b \rho) x=b\left(x^{b}\right)^{-1} \rho x
$$

the equality $b r=\left(b \rho_{z}\right)^{x}$ can be written as

$$
r=\left(x^{b}\right)^{-1} \rho_{z} x,
$$

where $r$ is any given element of the form (4), with the unknown elements $x=\left(x_{1}, x_{2}, \ldots, x_{n}\right) \in R$, and $\rho_{z}$ of the form (5). We shall find a solution where $\rho_{z}$ is of the form

$$
\rho_{z}=\left(p_{z}, e, e, \ldots, e\right) .
$$

Since $\left(x^{b}\right)^{-1}=\left(x_{n}^{-1}, x_{1}^{-1}, x_{2}^{-1}, \ldots, x_{n-1}^{-1}\right)$, the equation (6) is now:

$$
\left(p_{j_{1}}^{a_{1}}, p_{j_{2}}^{a_{2}}, \ldots, p_{j_{n}}^{a_{n}}\right)=\left(x_{n}^{-1}, x_{1}^{-1}, x_{2}^{-1}, \ldots, x_{n-1}^{-1}\right)\left(p_{z}, e, e, \ldots, e\right)\left(x_{1}, x_{2}, \ldots, x_{n}\right) .
$$

It implies $n$ equations on elements of $P$ with unknowns $p_{z}, x_{1}, x_{2}, \ldots, x_{n}$.

$$
p_{j_{1}}^{a_{1}}=x_{n}^{-1} p_{z} x_{1}, \quad p_{j_{2}}^{a_{2}}=x_{1}^{-1} x_{2}, \quad p_{j_{3}}^{a_{3}}=x_{2}^{-1} x_{3}, \ldots p_{j_{i}}^{a_{i}}=x_{n-1}^{-1} x_{n} .
$$

If multiply these equations by sides, we get

$$
p_{j_{1}}^{a_{1}} p_{j_{2}}^{a_{2}} p_{j_{3}}^{a_{3}} \cdots p_{j_{n}}^{a_{n}}=x_{n}^{-1} p_{z} x_{n}=p_{z}^{x_{n}} .
$$


Then, since $p_{j_{1}}^{a_{1}} p_{j_{2}}^{a_{2}} p_{j_{3}}^{a_{3}} \cdots p_{j_{n}}^{a_{n}}$ is an element in $P$, it is equal to some $p_{j}^{u}$, where $u \in R$. So we get the solution: $p_{z}=p_{j}$ and $x_{n}=u$. Then $\rho_{z}=\left(p_{j}, e, e, \ldots, e\right)$, and by $(8)$,

$$
\begin{gathered}
x_{1}=p_{j}^{-1} u p_{j_{1}}^{a_{1}}, \quad x_{2}=x_{1} p_{j_{2}}^{a_{2}}=p_{j}^{-1} u p_{j_{1}}^{a_{1}} p_{j_{2}}^{a_{2}}, \ldots, \\
x_{i}=x_{i-1} p_{j_{i}}^{a_{i}}=p_{j}^{-1} u p_{j_{1}}^{a_{1}} p_{j_{2}}^{a_{2}} \ldots p_{j_{i-1}}^{a_{i-1}} p_{j_{i}}^{a_{i}}, i<n .
\end{gathered}
$$

which finishes the proof.

Theorem 3.8. Let $P$ be an FNCC-group, $n \geq 1$, and $R=\prod_{i}^{n}{ }^{\times} P_{i}$, be the direct product of $n$ copies of $P$. Let $B \subseteq S_{n}$ be a group permuting factors in $R$. Then the semidirect product $G=R \rtimes B$ is an FNCCgroup.

Proof. Lemma 2.1(ii) applied to $G \subseteq R \rtimes S_{n}$ allows us to prove the result only for $B=S_{n}$. We are going to proceed by induction on $n$. For $n=1$ we have $G \simeq P$ and the result is trivial.

Now let $n>1$. If $\sigma \in S_{n}$ is a cycle of length $n$ then, by Lemma 3.7 , the coset $\sigma R$ is contained in a union of finite number of conjugacy classes. If $\sigma$ is not of such type then we can write $\sigma=\gamma \delta$, where $\gamma$ permutes cyclically $m<n$ factors of $R$ and is fixed on the others, while $\delta$ permutes at most $n-m$ factors of $R$, fixed by $\gamma$. Then $\gamma \subseteq S_{m}$ permutes factors of $P^{m}$ and $\delta \subseteq S_{n-m}$ permutes factors of $P^{n-m}$ in a natural way. Let $G_{1}$ be the semidirect product of $P^{m}$ with $\langle\gamma\rangle$ and $G_{2}$ the semidirect product of $P^{n-m}$ with $\langle\delta\rangle$ under these actions. Then, by Lemma 2.1(ii) applied to extensions $\left\langle P^{m}, \gamma\right\rangle \subseteq G_{1},\left\langle P^{n-m}, \delta\right\rangle \subseteq G_{2}$, and by the inductive assumption, $G_{1}$ and $G_{2}$ are $F N C C$-groups. Thus $G_{1} \times G_{2}$ is also an $F N C C$-group, contained in $G$ in a natural way. Moreover, $\sigma R \subseteq G_{1} \times G_{2}$. This means, that $\sigma R$ is contained in a union of a finite number of conjugacy classes in $G$. Now the result follows, because $R$ is of finite index in $G$.

For further text we recall that the restricted wreath product $A w r B$ of groups $A$ and $B$ is a semidirect product $G=R \rtimes B$ where $R=\prod_{b \in B}{ }^{\times} A^{b}$ is the direct product of copies of $A$, numbered by elements of $B$ and $B$ acts on $R$ by shifting indices. Instead of $A^{e}$ we write $A$, and $A^{b}=b^{-1} A b$. Every element $g \in G$ can be uniquely written as $g=b w$ where $b \in B$ and $w$ is a product of commuting factors $a^{b}$, where $b \in B$, $a^{b}=b^{-1} a b, \quad b a \cdot b_{1} a_{1}=b b_{1} a^{b_{1}} a_{1}$.

Now we give a criterion for a restricted wreath product of groups to be an $F N C C$-group.

Theorem 3.9. A restricted wreath product $A$ wr $B$ is an $F N C C$-group if and only if $A$ is an FNCC-group and $B$ is finite. 
Proof. Let $G=A$ wr $B=\left(\prod_{b \in B}^{\times} A^{b}\right) \rtimes B=R \rtimes B$ be an $F N C C$ group. In the restricted wreath product each element $r \in R$ has a finite support of the length $s(r)$, say. Moreover, the conjugate elements have supports of the same length. If $G$ is an $F N C C$-group, then the lengths of possible supports have only finite number of values, which is possible only if the group $B$ is finite. Then $R$ is a subgroup of finite index in $G$ and by Lemma 2.1(ii), $R$ is an FNCC-group. Then $A$ is an FNCC group as an image of $R$.

The converse implication follows from Theorem 3.8, because the group $B$ acts by permutations on the subscripts of direct factors in $R$.

\section{REFERENCES}

[1] R. G. Burns and Y. Medvedev, Group laws implying virtual nilpotence, J. Aust. Math. Soc. 74 (2003), 295-312.

[2] G. Cutolo, H. Smith and J. Wiegold, Groups covered by conjugates of proper subgroups, J. Algebra 293 (2005), 261-268.

[3] Yu.M. Gorchakov, Groups with finite classes of conjugate elements, Nauka, Moscow, 1978 (in Russian).

[4] Yu.N. Gorchinskii, Groups with a finie number of conjugacy classes, Mat. Sbornik N.S. 31 (1952), 167-182.

[5] Yu. N. Gorchinskii, Periodic groups with a finie number of conjugacy classes, Mat. Sbornik N.S. 31 (1952), 209-216.

[6] B. Hartley, Simple locally finite groups, in: Finite and Locally Finite Groups, Istanbul, 1994, NATO Adv. Sci. Inst. Ser.C Math. Phys. Sci., 471, Kluwer Academic, Dordrecht, 1995, 1-44.

[7] I.N. Herstein, Noncommutative rings, MAA, 1968.

[8] M. Herzog, P. Longobardi and M. Maj, On generalized FC-groups, J. Group Theory 11 (1) (2008), 105-117.

[9] G. Higman, B. H. Neumann and H. Neumann, Embedding theorems for groups. J. London Math. Soc. 24 (1949). 247-254.

[10] E. I. Khukhro and V. D. Mazurov (eds.), Unsolved problems in group theory, The Kourovka Notebook, no. 17, Novosibirsk, 2010.

[11] J. Krempa, On finite generation of unit groups for group rings, in: Groups '93 Galway/St. Andrews, Vol. 2, London Math. Soc. Lecture Note Ser., 212, Cambridge Univ. Press, Cambridge, 1995, 352-367.

[12] O. Macedońska and W. Tomaszewski, On Engel and positive laws, in: Groups St Andrews 2009 in Bath, Vol. 2, London Math. Soc. Lecture Note Ser., 388, Cambridge Univ. Press, Cambridge, 2011, 461-472.

[13] A. Yu. Ol'shanskii, Geometry of defining relations in groups, Kluwer Academic Publishers Group, 1991.

[14] D.V. Osin, Small cancellations over relatively hyperbolic groups and embedding theorems, Annals of Math. (2) 172(1) (2010), 1-39.

[15] D.J.S. Robinson, Finiteness conditions and generalized soluble groups Part I, Ergebnisse der Mathematik und ihrer Grenzgebiete v.62, Springer-Verlag, New York Heidelberg Berlin, 1972.

[16] D.J.S. Robinson, A course in the theory of groups, Springer-Verlag, New York Heilderberg Berlin, 1982.

[17] M. J. Tomkinson. FC-groups, Pitman, Boston MA, 1984. 
Jan Krempa, Institute of Mathematics, University of Warsaw, BaNACHA 2, 02-097 WarszaWa, Poland

E-mail address: jkrempa@mimuw.edu.pl

Olga Macedońska, Institute of Mathematics, Silesian University of Technology, Kaszubska 23, 44-100 Gliwice, Poland

E-mail address: 0lga.Macedonska@polsl.pl

Witold Tomaszewski, Institute of Mathematics, Silesian University of Technology, Kaszubska 23, 44-100 Gliwice, Poland

E-mail address: Witold.Tomaszewski@polsl.pl 